\title{
Visualisation of fingermarks and grab impressions on dark fabrics using silver vacuum metal deposition
}

Susan Knighting ${ }^{1}$ Joanna Fraser ${ }^{1}$, Keith Sturrock $^{1}$, Paul Deacon ${ }^{2}$, David H Bremner $^{1}$

${ }^{1}$ School of Contemporary Sciences, University of Abertay Dundee, Bell Street, Dundee DD1 1HG, UK

${ }^{2}$ Forensic Services, Scottish Police Services Authority, Rushton Court, 3 West Victoria Dock $R d, D D 1$

\section{Abstract}

Vacuum metal deposition (VMD) involves the thermal evaporation of metal (silver) in a vacuum, resulting in a uniform layer being deposited on the specimen being treated. This paper examines the use of silver on dark fabrics, thus offering a simpler operation and more obvious colouration to that of the traditional use of gold and zinc metals which must be evaporated separately. The aim of this study was to investigate the effect of fabric type, donor, mark age and method of fingermark deposition on the quality of marks visualised using silver VMD. This was achieved by collecting fingermark deposits from fifteen donors, of both sexes and various ages, by a grab or a press method. Four different fabrics: satin, polyester, polycotton and cotton were studied over a 10 day timeline of $1,2,3,4,5,6,7,14,21$ and $28+$ days. It was found that satin and polyester gave the most positive results, with polyester often producing excellent ridge detail. Cotton and polycotton were less successful with no ridge detail being observed. The donors also had a observable effect on the results obtained probably due to variations in secretions produced or pressures applied during specimen collection. The age of the mark or the method of mark deposition had little influence on the results obtained. Silver VMD is a viable process for visualising marks on certain dark fabrics and has the advantage over gold/zinc VMD in that the marks visualised are light in colour which contrasts well against the dark background. 
Keywords: Fingermarks; Silver; Vacuum metal deposition; Fabrics

\subsection{Introduction}

When someone places a hand on a surface, residues present on the skin are usually deposited, thus leaving an invisible or 'latent' mark. These residues are a mixture of secretions from eccrine, sebaceous and apocrine sweat glands and environmental contaminants such as, grease and dirt.

As latent marks cannot be seen by the naked eye they require either physical or chemical enhancement in order to make them visible and suitable for identification. The amount of residue produced by the sweat glands and thus the ability of a mark to be deposited onto a surface is affected by several factors such as temperature, food consumption, activity level, a person's age, gender and health and the nature of the surface itself. After deposition other factors become important, including the time elapsed since deposition and the environmental conditions to which the mark has been exposed. Thus different techniques are utilised for the enhancement of marks exposed to varying conditions [1]. In the case of chemical enhancement, the technique chosen is largely dependent upon the type of substrate (paper, metal, plastic or fabric etc) onto which the marks have been deposited. Substrates can be considered to be porous, semi-porous or non-porous depending on how easily mark constituents are absorbed [2] and in the UK, the most suitable method and sequence of enhancement can be determined from the Manual of Fingerprint Development Techniques (MFDT) [1].

Fabrics are considered to be 'difficult' (i.e. fingermark recovery from them is typically very low) substrates which do not have an established process for developing latent marks. In the UK it is recommended that the fabric must be clean and have at least three threads per $\mathrm{mm}$ of material in order to ensure that the mark residues are retained by the fabric [3]. Recently, we reported on the use of gold/zinc vacuum metal deposition for the acquisition of fingermarks and grab impression on fabrics and it was concluded that greater ridge detail was visible on smooth and less porous fabrics [4]. We now describe our results obtained 
from the use of silver VMD and highlight the advantages of using this technique on dark fabrics.

VMD, which was originally introduced as an industrial tool for applying metal coatings to objects such as mirrors [5] provides a means of treating items that have been exposed to adverse environmental conditions since VMD can detect sebaceous material in the mark even if the substrate has been wet [6]. Latent marks deposited on a variety of non-porous surfaces, such as polythene carrier bags as well as some semi-porous substrates, can be enhanced using VMD which works by evaporating and depositing a thin layer of metal or a combination/sequence of metals onto the specimen within a vacuum chamber [5-11].

As well as these non-porous surfaces, some work has been performed using VMD on porous surfaces like paper [11] and fabric $[4,5,13]$ with some success. Silver is one metal suggested for use to enhance marks further after gold/zinc deposition [14]. Unlike gold/zinc, silver produces a uniform density coating on the substrate and also on the ridges. However, a different colour is observed on each of these regions due to the formation of silver clusters of different sizes and distributions and this makes the ridges distinct from the substrate background, making the mark more visible on the substrate, particularly, when compared to the dark grey colouration of the gold/zinc method. Furthermore, the silver VMD technique is much simpler to operate, requiring only the evaporation of one metal during the process. However, marks enhanced with silver are very fragile as the silver coating can easily be dislodged, so the mark must be handled with care to minimise the loss of any ridge detail. Also, as the Home Office Fingerprint Manual states, that since the marks may fade with time they must be photographed as soon as possible after treatment [14].

The current study investigated the potential of using silver as an alternative metal for VMD to enhance latent marks on a selection of darkcoloured fabrics so as to achieve a more obvious colour contrast and enable any detail to be more easily seen and recorded. An additional objective was to, assess whether or not the fabric type, donor, age and method of deposition had an effect on the quality of the marks obtained. 
Visualisation of fingermarks, along with any "press" or "grab" marks and consequently identifying areas to target for DNA taping might aid crime investigation.

\subsection{Materials and methods}

The fabrics (specimen size: $16 \mathrm{~cm} \times 23 \mathrm{~cm}$;) investigated were black polycotton $(60 \%$ cotton and $40 \%$ polyester mix), black polyester and black satin and dark blue cotton, which all had four threads per $\mathrm{mm}$ of material and were hand washed prior to use using detergent powder to remove any potential contaminants. Once prepared each fabric specimen was stored in a plastic wallet labelled with the donor number and day on which the marks were processed. There were fifteen male and female donors of different ages in the study, and all donated natural marks obtained at least 30 minutes after hand washing. All donors had been previously graded on substrates such as glass and paper thus indicating their relative ability to leave marks on these substrates.

The left hand grab mark was acquired by placing the fabric over the collector's arm and the donor grabbed it firmly for 5 seconds. The right hand was used for the press which was again obtained by laying the fabric on the collector's arm and having the donors press their fingers briefly on the fabric to simulate a push. After collection, the specimen was returned to its wallet until it was of the desired age, from 1 to $7,14,21$ or $28+$ days old, to be treated with Ag-VMD. Overall, this led to each donor donating 80 specimens in total - 10 days $\times 4$ fabrics $\times 2$ modes (grab and push). For each session a donor would only plant a grab from one hand and a push from the other on a single fabric specimen, the next specimen being collected on a separate day or after a minimum of at least an hour later, thus allowing their secretions to be replenished.

An Edwards' 24" metal deposition machine (Mason Vactron Ltd, UK) was used for the treatment of specimens. The silver wire $(0.5 \mathrm{~mm}$ in diameter 99\%; Sigma-Aldrich UK) was cut to approximately $5 \mathrm{~mm}$ lengths and 3 pieces $(30 \mathrm{mg}$ ) placed in the central evaporation boat within the VMD chamber [14]. The fabric specimen was placed in the chamber and it was evacuated to a pressure of $3 \times 10^{-1}$ mbar using rotary pumps at which point the system was switched to diffusion pumping to obtain a high 
vacuum. When the pressure in the chamber was reduced to about $4 \times 10^{-4}$ mbar, the current to the boat containing the silver was switched on and increased appropriately until the silver evaporated (about $5 \mathrm{sec}$ ). Test pieces of paper with pre-loaded fingermarks were placed next to the fabrics in the VMD chamber in order to confirm that the process was working properly. After treatment, the VMD chamber was brought back up to atmospheric pressure, the specimen removed, labelled with details of the fabric type, donor and test day and then photographed.

A scale-ruler was introduced and photographs of every specimen were taken immediately after removal from the chamber, using white light and oblique lighting as sources. The visualised marks were then graded, from "No development" to "Excellent", depending on the amount of ridge detail observed.

(0) No development - no visible or recognisable marks on fabric

(1) "Empty" marks - where the donor had touched the fabric could be seen but no ridge detail observed on fingertips or palm.

(2) Fair - Pattern and ridge flow and/or palmar flexion creases visible, but not enough detail for identification.

(3) Good - Ridge characteristics (Galton details) visible on some fingermarks.

(4) Excellent - good ridge detail on all fingertips and palm with visible pores, ridge edge detail and ridge flow.

\subsection{Results and discussion}

\subsection{Fabrics}

Fig. 1 (grab marks) shows the "quality values" for each fabric and was obtained by using a simple numerical calculation, where the number of marks is multipled by the quality of the mark (grades 1-4). Results show: cotton (22 marks grade 1 or higher), polycotton (45), polyester (138) and satin (58). Thus the results of this study clearly indicate that of the four fabrics examined, polyester afforded the highest number indicating that this was the best fabric from which to obtain marks and 
was the only fabric to produce grades higher than 1 (visible touch but no detail). The other fabrics - cotton, polycotton and satin only ever produced a maximum grade of 1 . The same trend is observed for press marks (Fig. 2) with cotton (40), polycotton (75), polyester (218) and satin (85). This high value for polyester is a result of it being the only fabric to show grades 2, 3 and 4.

The higher number of positive marks obtained from polyester is believed to result from the smooth, shiny, less porous tight weave of the fabric which helps retain the fingermark deposit [8]. In addition, since the fabric was washed prior to fingermarks being deposited, the plasticised 'waxy' layer that coats the material and which can make the VMD procedure more difficult, will have been eliminated [6]. Surface texture could account for variation in the observation of ridge detail. In satin it could simply be that the fabric is too shiny, hence obscuring any potential print detail whereas with cotton and polycotton, each fabric had a looser weave and rougher texture than the synthetic materials and therefore did not allow the fingermark residues to remain on the surface of the fabric but were absorbed. Generally, the quality of marks deposited on synthetics is much better than those deposited on natural fabrics when enhanced using Ag-VMD and it can be concluded that fabric type and thread count have major influences on the quality of marks obtained.

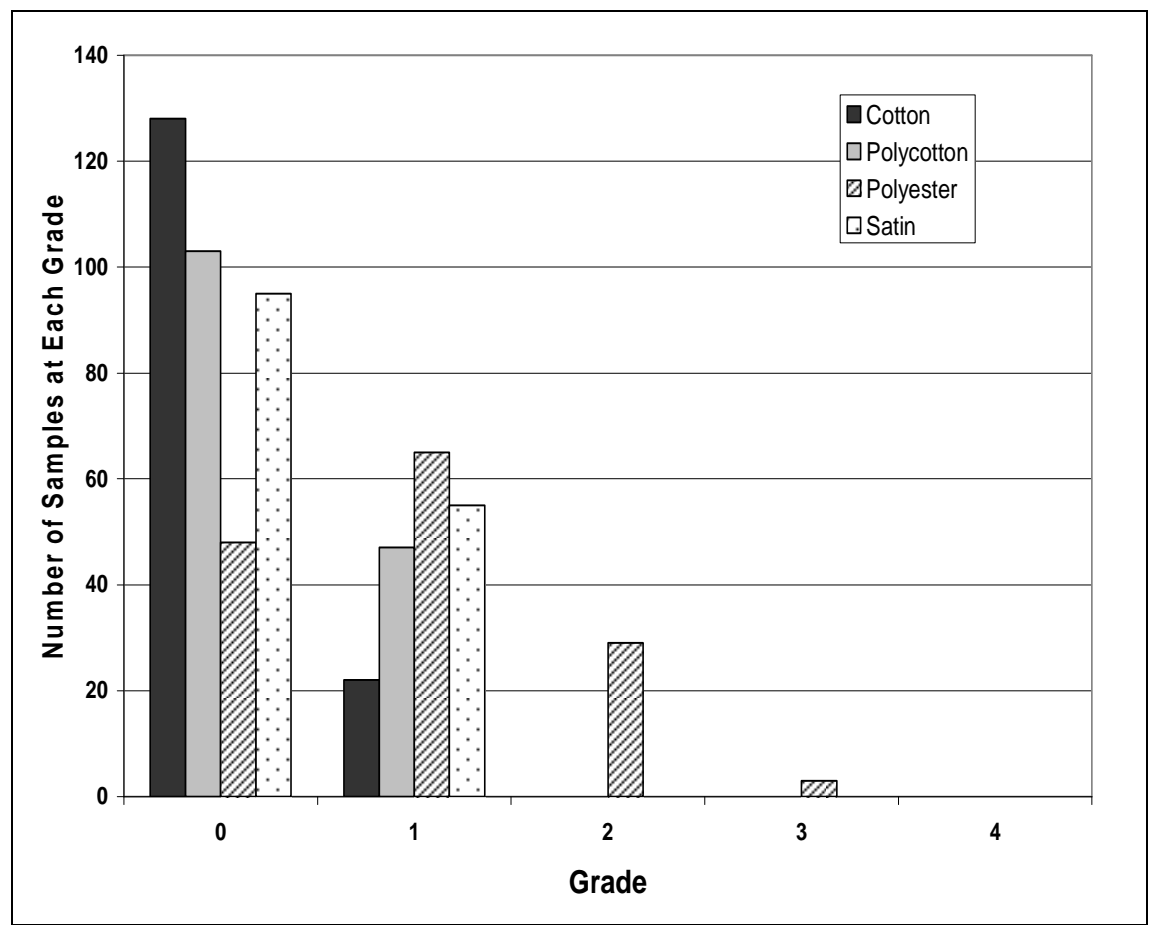


Fig. 1. Number of grab marks on cotton, polycotton, polyester \& satin specimens (graded 0-4).

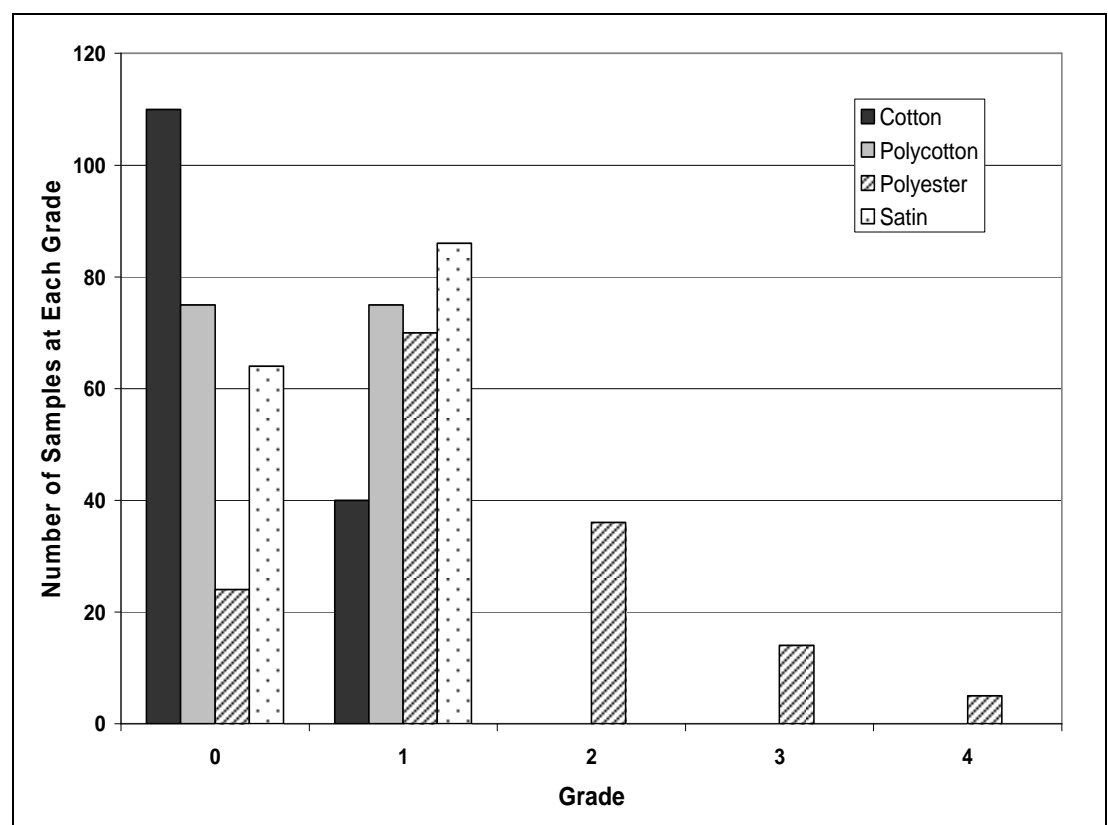

Fig. 2. Number of press marks on cotton, polycotton, polyester \& satin specimens (graded 0-4).

\subsection{Donor}

In this study all donors deposited in total 80 marks consisting of 10 different days on four fabrics using two methods (push and grab). Data from all the donors has been included in the analysis. The fingermark donor was also shown to have a considerable effect on the quality of marks enhanced on each of the fabrics. Each donor was graded before the experiment began in terms of good, medium or bad by acquiring fingermarks on paper and glass using black magna powder and aluminium powder respectively. Not surprisingly the pre-grading roughly mirrored the data shown below for the best fabric but the marks on paper were much more prevalent. However, occasionally a good donor on glass would give a poor mark on fabric whilst the opposite was also observed. Table 1 shows the gradings ascribed to each donor based on the number of days (10) multiplied by the number of fabrics (4) and the values for the 
grab marks + values for the push marks (8). A "perfect donor" would thus have a grading value of $10 \times 4 \times 8=320$.

\begin{tabular}{|l|l|l|l|l|l|l|l|l|l|l|l|l|l|l|l|}
\hline & \multicolumn{11}{c|}{ Donor Number } \\
\hline \multirow{3}{*}{ Total } & 1 & 2 & 3 & 4 & 5 & 6 & 7 & 8 & 9 & 10 & 11 & 12 & 13 & 14 & 15 \\
\cline { 2 - 26 } & 96 & 93 & 30 & 39 & 52 & 25 & 10 & 21 & 41 & 34 & 53 & 37 & 47 & 78 & 23 \\
\hline
\end{tabular}

Table 1. Total grading for each donor based on the number of fabrics multiplied by the number of days multiplied by the grade values allocated

Overall, the best donor was 1 (96), who showed press and grab marks on polyester of grades of 3 and above on days 1, 2, 4, 5, 6 and 21 and, at least, grade 1 marks were noted on all other days for all the fabrics, with the exception of days 6, 21 and 28 for cotton (Fig. 3). Donor 11 (53) is classed as a medium donor showing values greater than 2 for days $1,2,14$ and 28 . Contrast this with poor donor 7 (10) who produced marks on polyester and satin on only five of the days.

The fingermark donor had a considerable effect on the quality of marks enhanced on each of the fabrics. For example, grades of 3 for polyester were achieved on day 1 for donor 1 , whereas donors 7, 8 and 9 produced grades of zero. Similarly, some donors produced a visible result (grades 1 to 4 ) on all four fabrics treated on the same day compared to other donors who produced a visible result on perhaps only one or two of the fabrics (data not shown).

Such differences between the donors could be explained by a number of reasons. The prior activity level of the donor and the temperature of the environment when collecting the marks would affect the amount of perspiration produced and hence the amount deposited on the fabrics. Also, the natural secretions produced by each donor will vary. Alternatively, variations may have arisen between donors as a result of the collection procedure in which slightly different pressures may have been applied when depositing marks. This is supported by previous work which investigated the composition of latent mark residues and concluded that despite controlled measures being taken to keep the collection method the same, variations between donors occurred as a result of 
pressure differences and an unequal distribution of residues on the fingers [15]. Furthermore, as in the study by Jones et al. [9], it was identified that different amounts of gold metal were required for VMD depending on the plastic/polymer type as well as the thickness of the fingermark residue. Therefore, whereas some of the donors in this study may have deposited heavy marks requiring the optimum amount of silver metal to be used, marks deposited by other donors may have been lighter, hence requiring less silver for visualisation. This difference between donors and their ability to doposit secretions onto fabric is an area that requires further study.

In order to clearly show the different qualities of marks produced by varying donors over the ten treatment days, the results from good, medium and poor donors are illustrated in Figs. 3-5. In the case of a good donor (1), it can be seen that the area on the fabric touched was always visible on polyester, polycotton and satin, and a grade of 0 never being obtained with these fabrics while the only grade 0 results obtained were on days $6,21 \& 28$ for cotton (Fig. 3). Donor 11 can be classed as a medium donor due to showing a grade of 2 or above on only four days on polyester. However, marks of a lower value were seen on all specimens of polyester for this donor. Once more, cotton proved to be the poorest fabric for giving marks with only three days showing just grade 1 (Fig. 4). Donor 7 (Fig. 5) produced consistently poor results on all fabrics. In fact, the area that had been touched was not visible on any cotton or polycotton specimens and, a grade of 2 was seen on only one specimen (polyester; day 4) and grades of 1 were only observed on a total of four times (day 1, 5, 7 and 14) for polyester and on satin four times (day 1, 4, $5 \& 7)$ throughout the entire study.

It was interesting to note that the donors' ability to provide visible marks was variable. Some donors were classed as good for glass yet provided poor results on fabrics. Also good donors on fabrics did not always provide high gradings and the values quoted above are an average snapshot of donors' abilities on fabrics. Thus caution needs to be applied when trying to predict the ability of a donor to provide identifiable marks on fabrics. Since the donor's abilities were variable and not enough specimens were available no statistical analysis was attempted. 


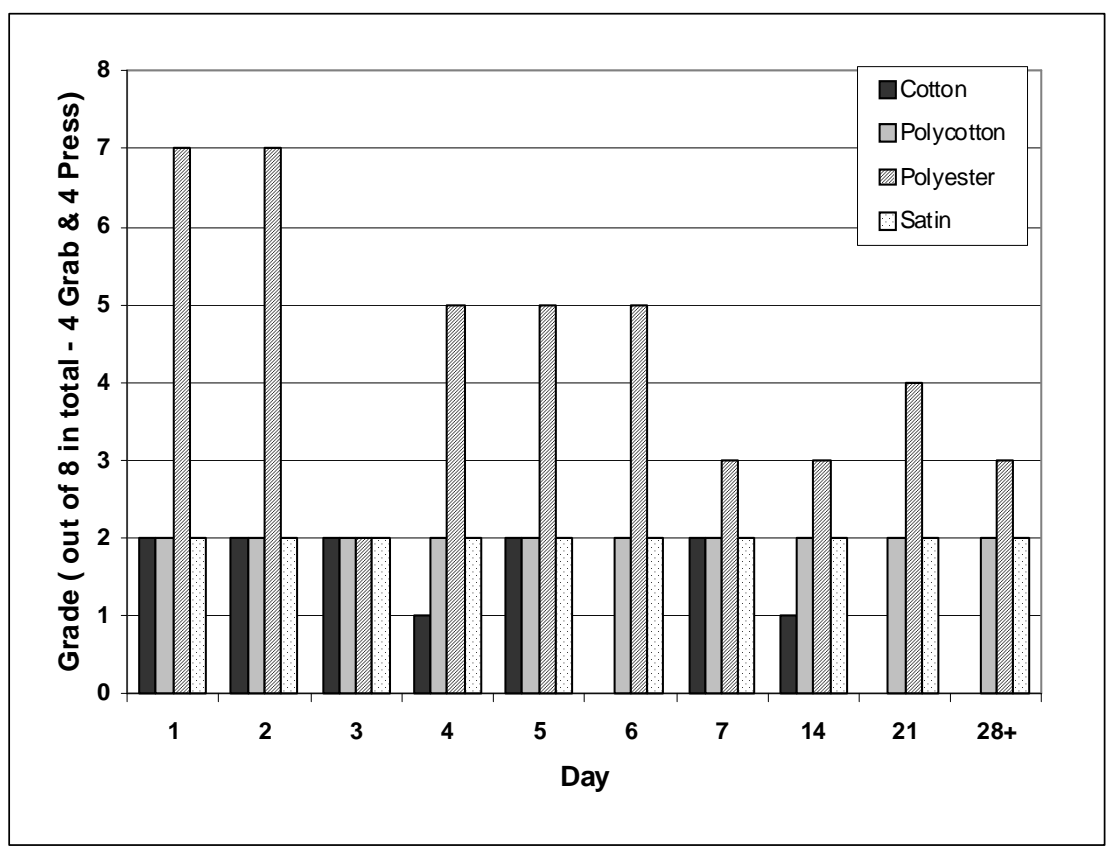

Fig. 3. Combined values of press and grab results for a good donor (Donor 1) on fabrics: cotton, polycotton, polyester \& satin.

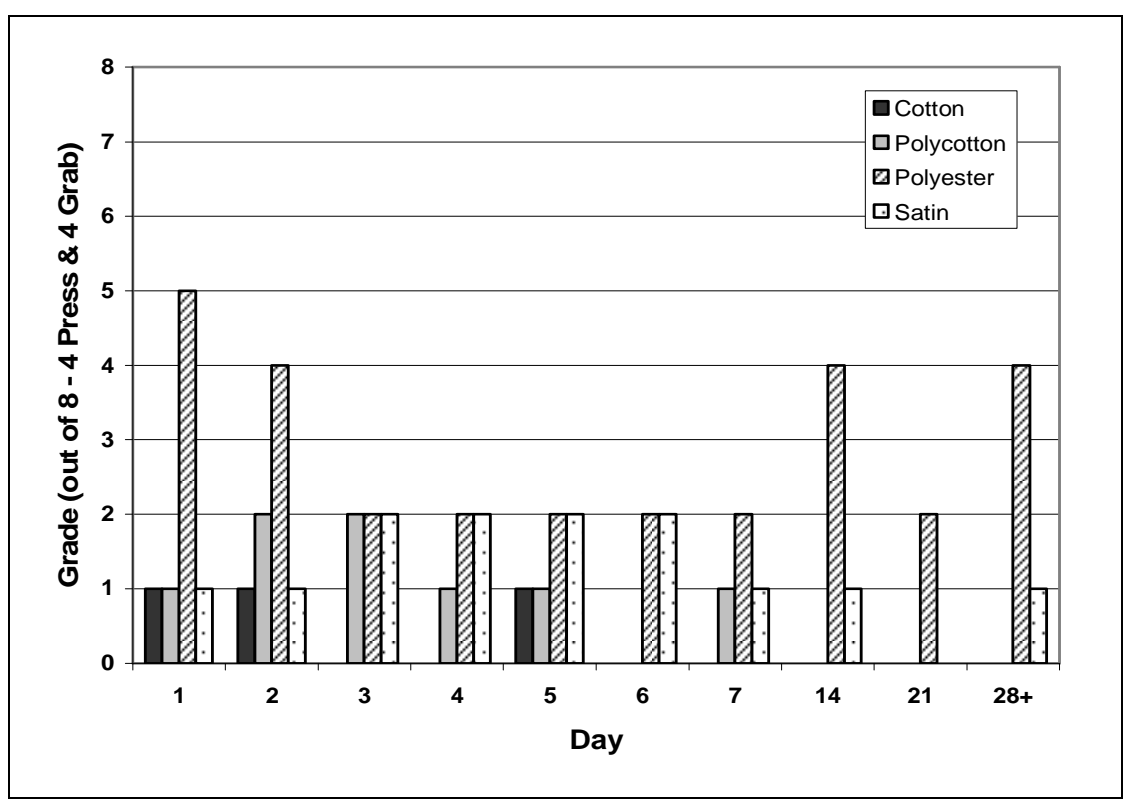

Fig. 4. Combined values of press and grab results for a medium donor (Donor 11) on fabrics: cotton, polycotton, polyester \& satin. 


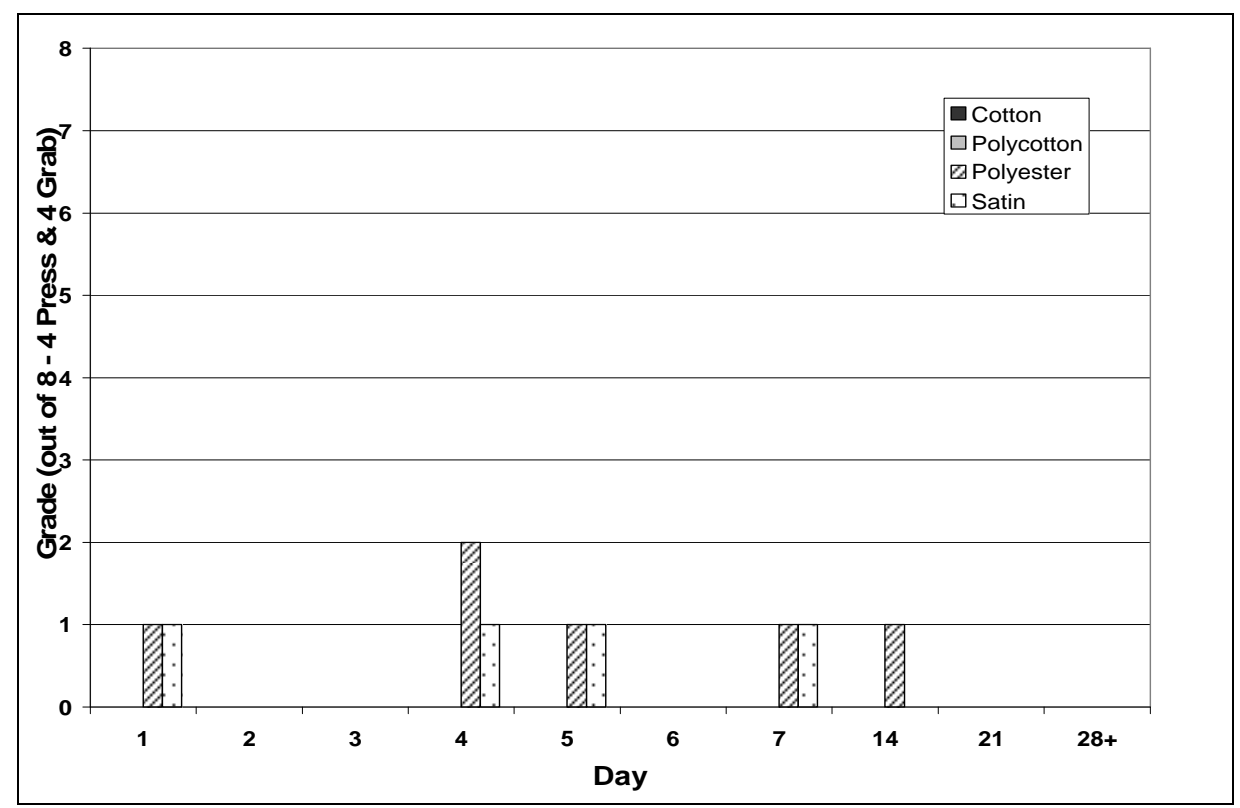

Fig. 5. Combined values of press and grab results for a poor donor (Donor 7) on fabrics: cotton, polycotton, polyester \& satin.

\subsection{Age of fingermarks}

Unlike fabric type and donor, the age of fingermark deposits did not have a evident effect on the quality of marks developed and Figs. 6 and 7 clearly illustrate this, showing the combined results from every donor over the ten days. For instance, a total of 23 'no touches' were recorded on day 1 for cotton (Fig. 6) with a visible result only being achieved by seven donors. The number of 'no touch' grades then fluctuated for the remaining treatment days, indicating that no correlation existed between age of mark deposits and quality of enhanced marks. The best results for cotton, albeit very poor, were seen on days 2 and 7. Marks that were 7 and 14 days old gave the best results on the polycotton, but none of them were above a grade 1 . With satin the days could be ranked, from most to least number of 'touches', in the order of day $1,7,4,5 / 6$; once more there is no obvious correlation between age of specimen and quality of the mark obtained. Of all the fabrics studied, polyester provided the highest quality and number of marks and the best results were achieved on day 1 with pores even being visible for four of the donors (Fig. 7). 


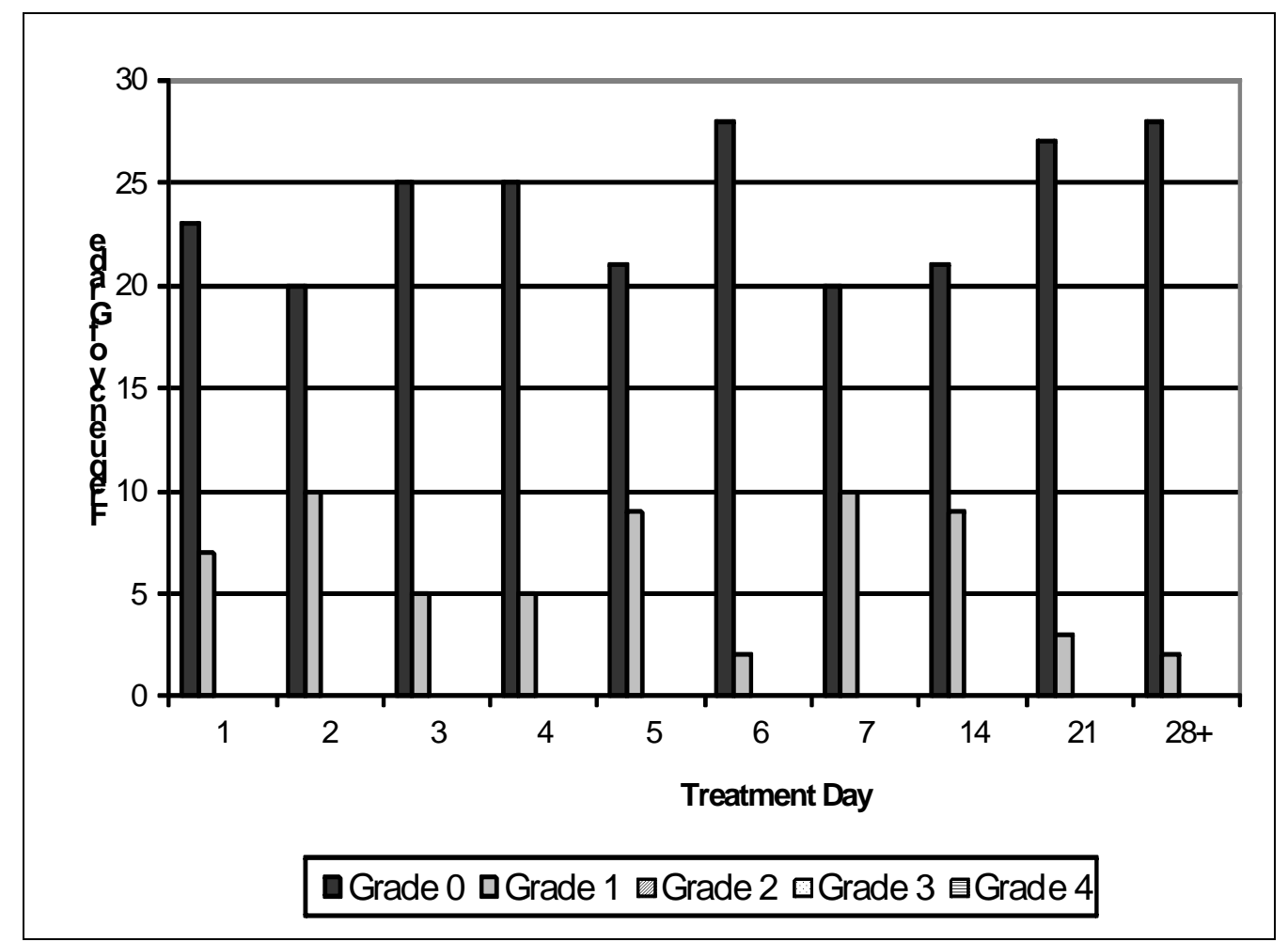

Fig. 6. Quality of marks produced each day on cotton using combined results for the grab and press methods of depositing impressions.

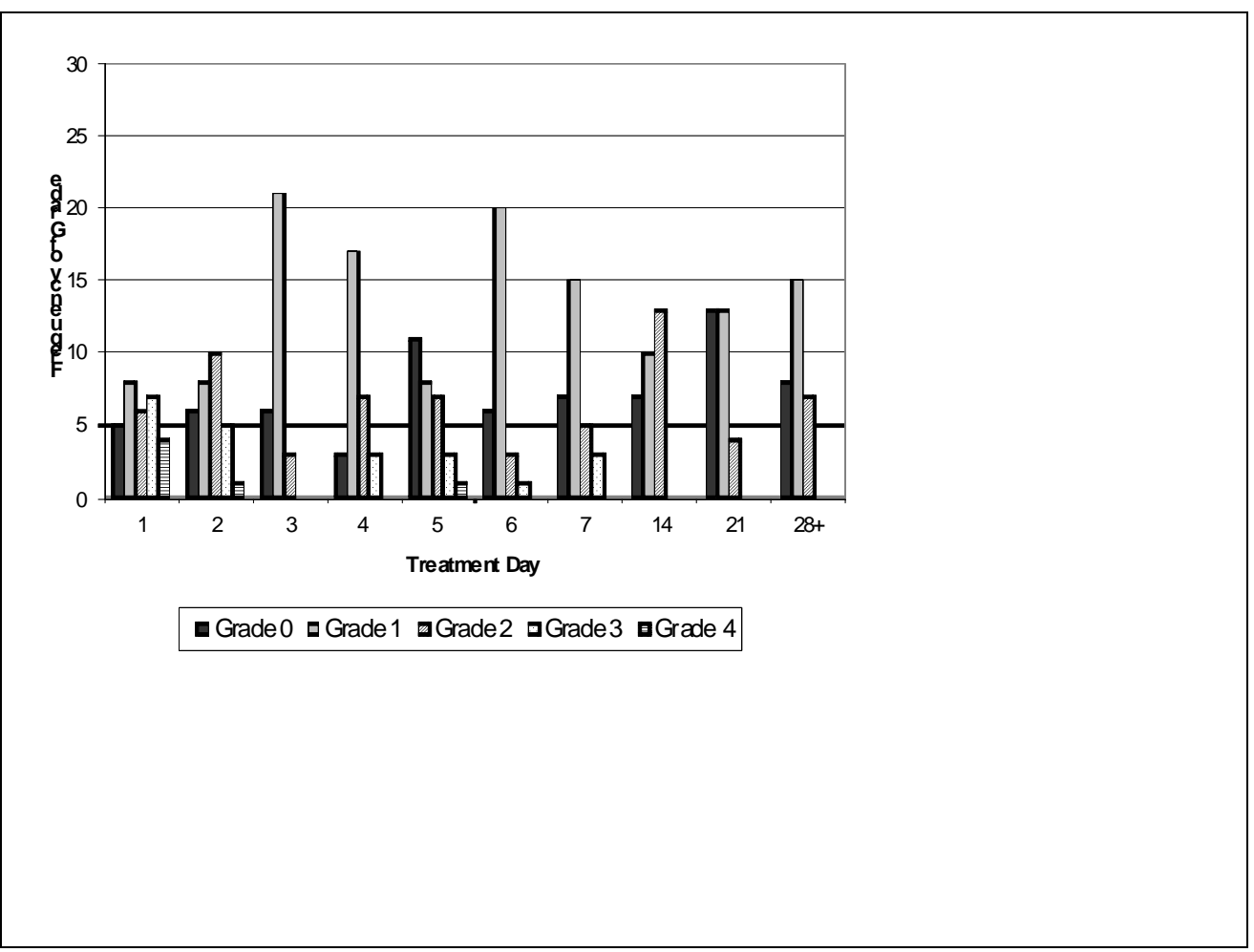

Fig. 7. Quality of marks produced each day on polyester using combined results for the grab and press methods of depositing impressions 
Although it would appear that there is, generally, little difference in detail from day 1 to day 28 this may be ascribed to the way in which the specimens were stored rather than inherent stability of the marks. Further work will be carried out to establish if air drying, plastic folders or paper storage would produce different results. One interesting observation was made during the study when two of the specimens were re-analysed 5 days after being treated with VMD. It was noticed that these specimens, which had achieved grades of zero, had now improved so much so that the area touched was now visible and in one instance, even ridge detail was observed. This suggests that after VMD treatment, it may be beneficial to re-examine specimens as marks may continue to "develop" over time giving improvement in contrast, probably due to oxidation of the silver deposit. Further work in this area needs to be done to see if this is a general phenomenon.

\subsection{Method of fingermark deposition}

The method of fingermark deposition did have a slight effect on the calibre of marks produced, with superior quality generally arising from the pressing action rather than grabbing. Fig. 8 gives a comparison between the two methods and clearly shows that overall, the press method produced more marks than the grab (18 - 31 more marks per fabric). In some instances the difference between the two methods was quite noticeable for some donors; for example donor 5 achieved a grade 2 on the polyester press, yet the area touched was not visible on the grab. Another example of differing results on the same fabric specimen was observed for donor 13 on day 5 where grades of 3 and 1 were attained for the press and grab, respectively. In many cases there was actually little variation between the press and grab actions, the same donor producing equally good results (or bad) for both (data not shown).

The differences that arise from the modes of deposition could potentially be useful forensically as the marks, be they grab or push, appear visually different - the fingers in the pushes appear straighter and 
thinner when compared to the curved and wider grab marks. Therefore this may be an indication of the method(s) used during an attack.

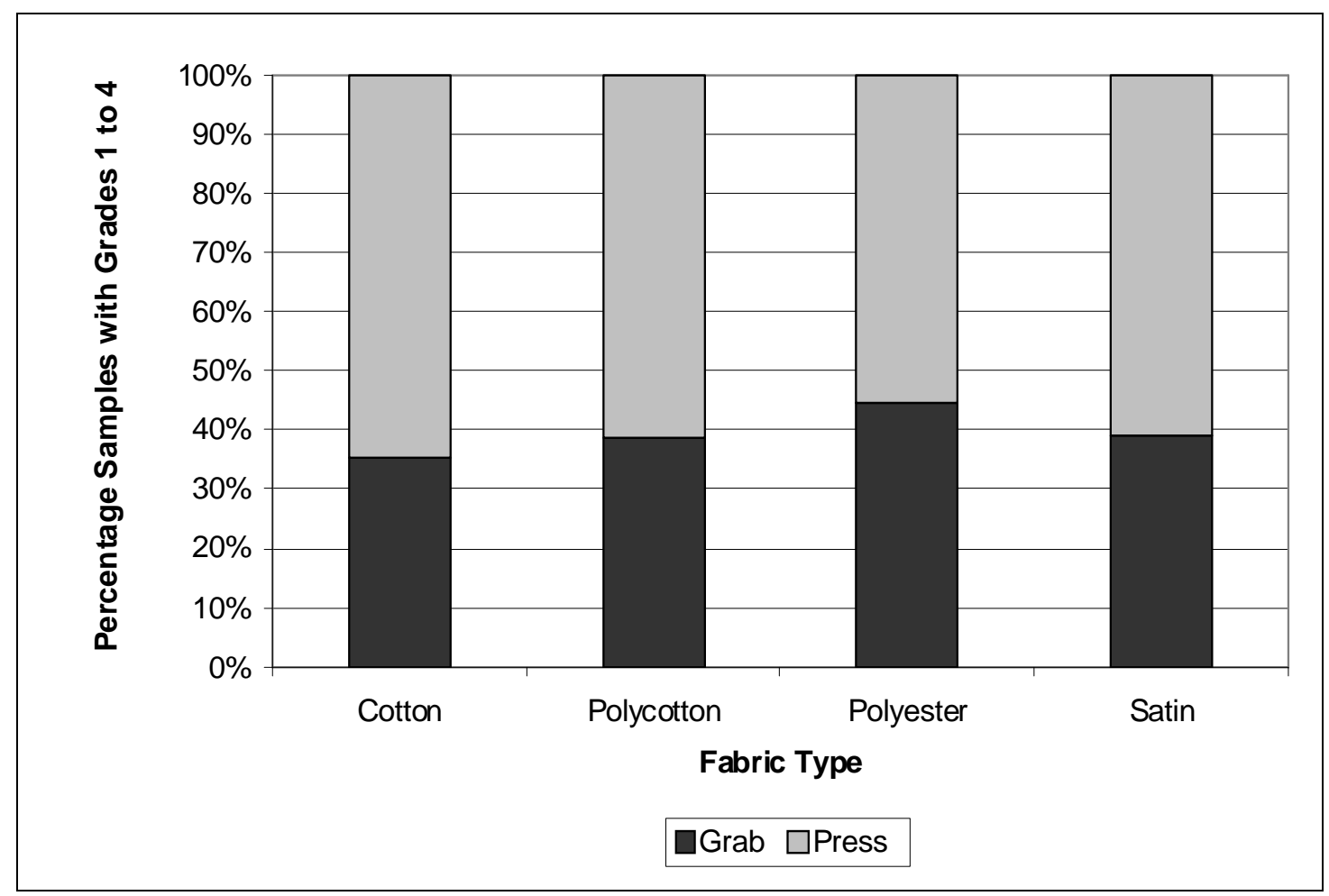

Fig. 8. Comparison of grab versus press depositing of impressions.

Another difference was that whereas the grab usually provided good detail in the palm area, the press tended to produce better ridge detail in the fingertips (Figs. 9. and 10). This is a likely consequence of the different pressures applied for each method during collection. For example, with the press, the hand was pushed onto the fabric with a constant pressure, depositing residues more evenly from all fingertips. Conversely, with the grab, an uneven pressure would be applied with less pressure at the fingertips, some fingers touching the fabric more firmly than others.

This study was an initial investigation into the acquisition of fingermarks from dark fabrics using Silver metal VMD. It is recognised that no depletion studies were carried out and consequently the sensitivity of the technique has not been examined. 


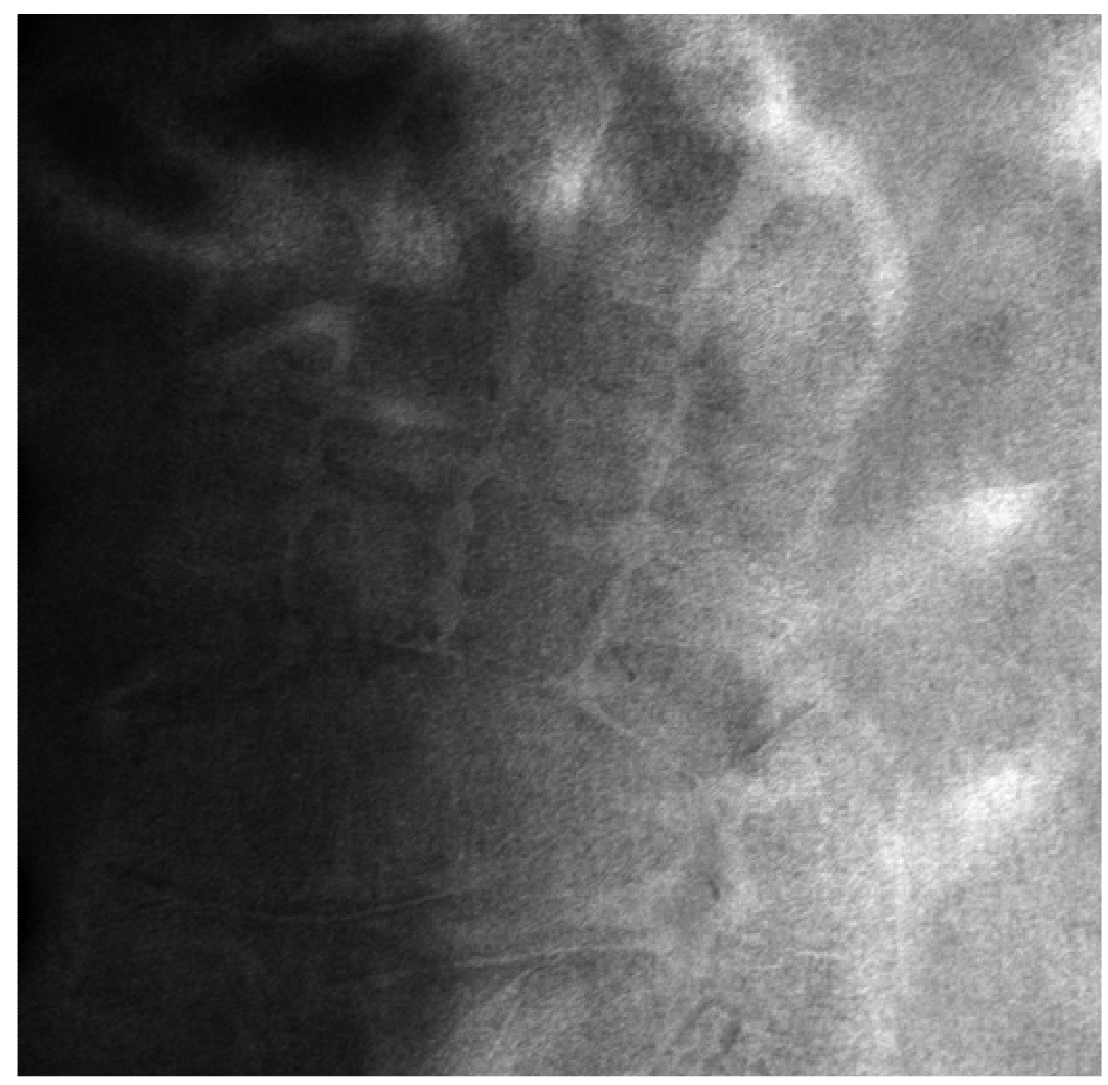

Fig. 9. Palmar flexion crease detail - deposited as a grab on polyester fabric, achieveing a grade of 2

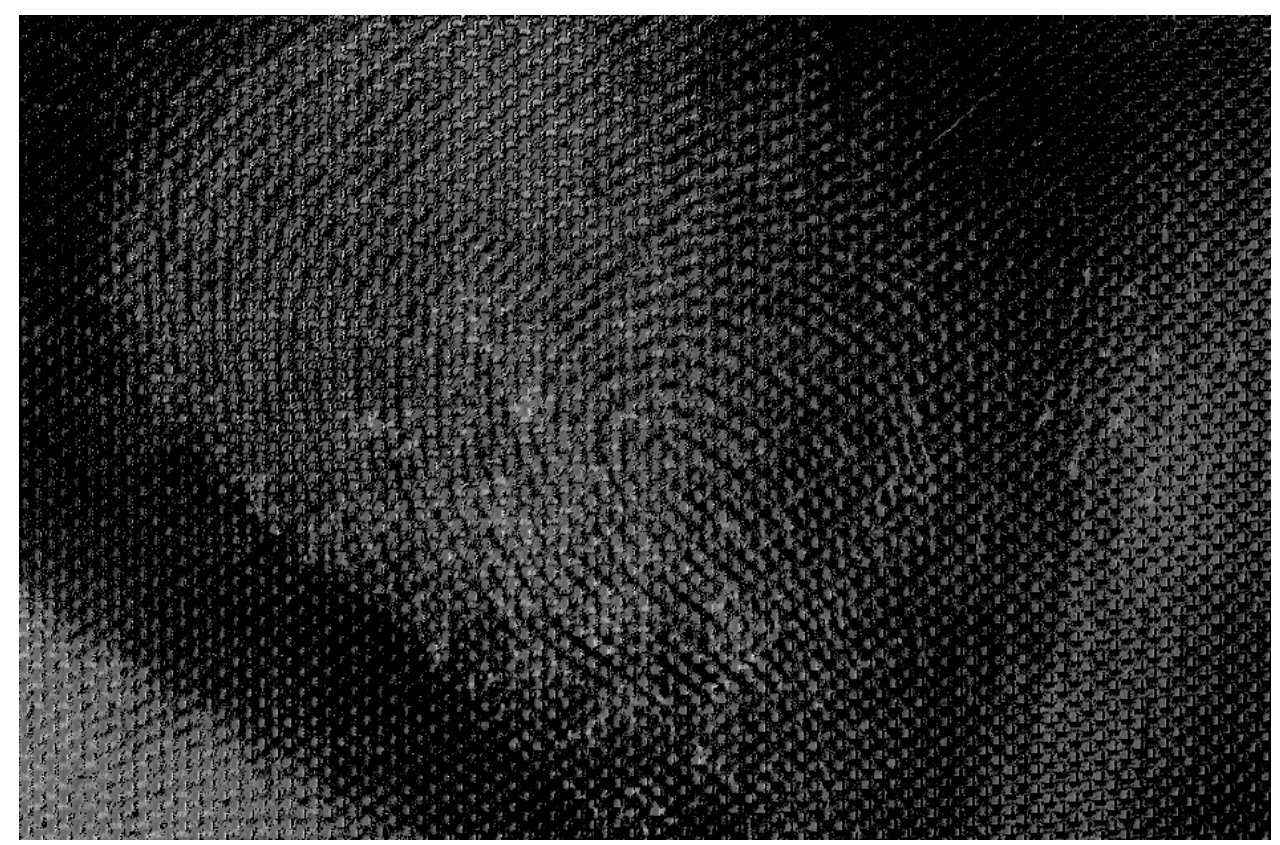


Fig. 10. Enhanced fingermark on polyester grab (Donor 1, Day 14), illustrating ridge detail.

\subsection{Visualisation of empty marks}

As illustrated in Fig. 11, some specimens show areas with no ridge detail and only outlines of marks (empty marks). However, these areas can still give indications of where a specimen has been touched, therefore even though no identification could be made via ridge detail these highlight areas from which DNA samples might be obtained and an identification of an individual could be attempted via this approach [16]. Polyester produced the most target areas, from $57 \%$ (on day 21 ) to $90 \%$ (on day 4), whereas cotton was the least succesful only producing target areas of $7 \%$ (on day 6 and 28) to 33\% (on day 2 and 7). Polycotton and satin both produced similar numbers of target areas with satin generally producing more than polycotton with the exception of days $2,3 \& 14$ (Fig. 12). This does demonstrate the point that even if no ridge detail is visualised, Ag-VMD treatment of the specimen is still of use as it will readily indicate areas for DNA taping.

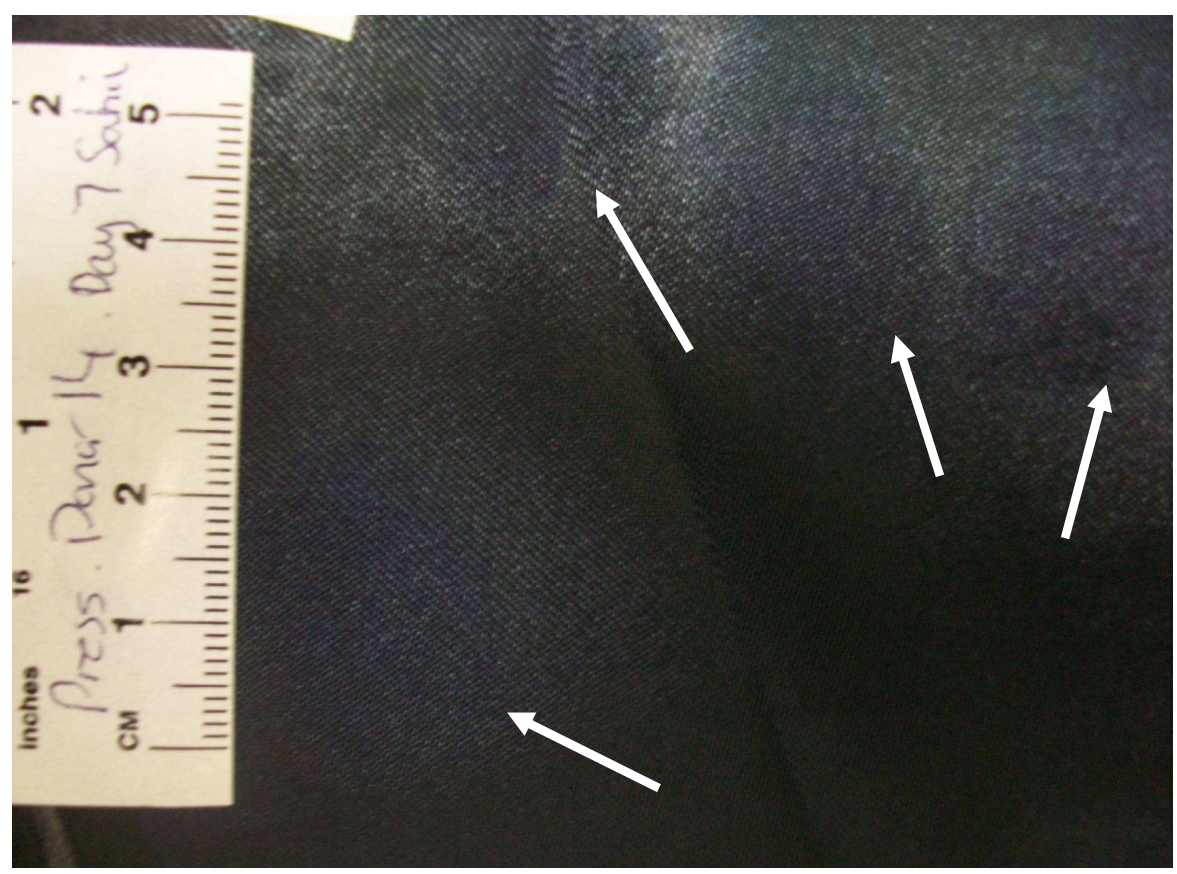

Fig. 11. - Day 7, Donor 14 "empty" marks on satin press as indicated by arrows. 


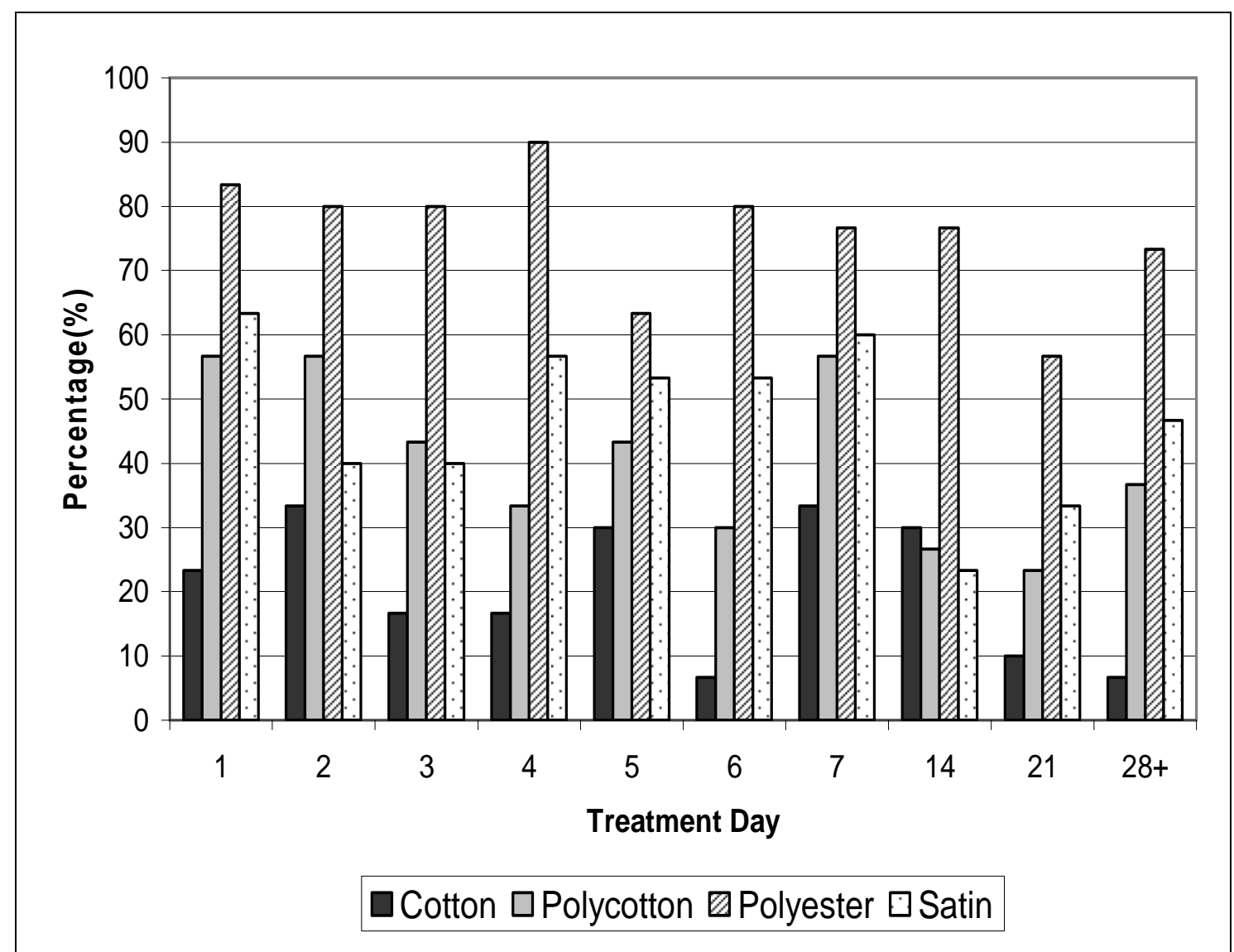

Fig. 12. Target areas visualised on the fabric specimens (cotton, nylon, polyester, polycotton and satin), indicating areas that could be taped for DNA.

\section{Conclusions}

The deposition of silver onto dark fabric specimens proved to be effective and, in the case of polyester, areas of ridge detail were developed. Where no ridge detail was seen (cotton, polycotton and satin) it could still be ascertained that the fabric had been touched. The donor had a major effect on the results, probably due to differences in secretions produced or pressures applied during specimen collection. The age of the deposits did not affect the quality of marks developed with the best results being obtained on day one though some donors produced good results with older marks. Although the 'press' method of fingermark deposition was shown to be superior (better detail in the fingermarks) for most of the specimens treated, the grab also produced valuable information. The main benefit of using silver rather than the traditional gold/zinc is that it is easier to see ridge detail and target areas on dark 
fabric and with this technique an assessment could be made about the way the fabric had been touched, either by pressing or grabbing and, even if an identifieable mark is not obtained, an area for DNA acquisition can be identified [16].

\section{Acknowledgements}

The authors would like to thank all the donors who participated in the project for the time spent during fingermark collections and also to members of the SPSA Dundee fingerprint laboratory team for their expertise and assistance.

\section{References}

[1] V. Bowman. (ed.), Manual of fingerprint development techniques, $2^{\text {nd }}$ ed, White Crescent Press Ltd., Luton, 1998. ( $3^{\text {rd }}$ revision, December 2009)

[2] C. Champod, C. Lennard, P. Margot ,M. Stoilovic, Fingerprints and Other Ridge Skin Impressions, CRC Press, BocaRaton,2004, pp. 236.

[3] D. S. Hambley, The physics of vacuum evaporation development of latent finger prints, PhD Thesis, Department of Physics, The Royal Holloway College, March, 1972.

[4] J. Fraser, K. Sturrock, P. Deacon, S. Bleay, D. H. Bremner, Visualisation of fingermarks and grab impressions on fabrics. Part 1: Gold/zinc vacuum metal deposition, Forensic Sci. Int. 208 (2010) 74-78.

[5] D. Philipson, S. Bleay, Alternative metal processes for vacuum metal deposition. J. Forensic Ident. 57 (2007) 252-273.

[6] E. Palma, Friction skin impressions. In: R. H. Walton, ed. Cold case homicides: practical investigative techniques. CRC Press. 2006, 293-296.

[7] T. Kent, G.L. Thomas, T.E. Reynoldson, H.W. East, Vacuum coating technique for development of latent fingerprints on polythene, J. For. Sci. Soc., (1976) 93-101. 
[8] A. H. Misner, Fingerprint detection using vacuum metal deposition, Canadian Police Research Centre, TM-15-93, November 1993

[9] N. Jones, D. Mansour, M. Stoilovic, C. Lennard, C. Roux, The influence of polymer type, print donor and age on the quality of fingerprints developed on plastic substrates using vacuum metal deposition. Forensic Sci. Int. 124 (2001) 167-176.

[10] N. Jones, M. Stoilovic, C. Lennard, C. Roux, Vacuum metal deposition: developing latent fingerprints on polyethylene substrates after deposition of excess gold, Forensic Sci. Int. 123 (2001) 5-12.

[11] X. Dai, M. Stoilovic, C. Lennard, N. Speers, Vacuum metal deposition: visualisation of gold agglomerates using TEM imaging, Forensic Sci. Int. 168 (2006) 219-222.

[12] H. C. Lee, R.E. Gaensslen, Methods of latent fingerprint development. In: Lee, H. C and Gaensslen, R. E, eds. Advances in fingerprint technology. $2^{\text {nd }}$ ed. CRC Press. 2001. p. 36.

[13] Home Office Scientific Development Branch (HOSDB). Treatment of fabrics. Fingerprint Development and Imaging Newsletter, April 2005. Publication No. 20/05.

[14] Home Office Scientific Development Branch (HOSDB), Silver VMD, Fingerprint Development and Imaging Newsletter, October 2005. Publication No. 47/05.

[15] N. E. Archer, Y. Charles, J. A. Elliott, S. Jickells, Changes in the lipid composition of latent fingerprint residue with time after deposition on a surface. Forensic Sci. Int. 154 (2005) 224-239.

[16] Personal Communication from Ignacio Quinones. Initial studies have been carried out on acquiring DNA from fingermarks visualised after VMD treatment on fabric swatches, T-shirts as well as chemises and indicates that it is possible to obtain full DNA profiles - this work is currently in the process of being submitted for publication. 